Nouvelles perspectives en sciences sociales

\title{
Entre représentations hiérarchiques et pratiques complexes : les paradoxes de la décentralisation française
}

\section{Pascal Roggero}

Volume 1, numéro 1, mars 2005

URI : https://id.erudit.org/iderudit/602446ar

DOI : https://doi.org/10.7202/602446ar

Aller au sommaire du numéro

Éditeur(s)

Prise de parole

\section{ISSN}

1712-8307 (imprimé)

1918-7475 (numérique)

Découvrir la revue

\section{Citer cet article}

Roggero, P. (2005). Entre représentations hiérarchiques et pratiques complexes : les paradoxes de la décentralisation française. Nouvelles perspectives en sciences sociales, 1(1), 63-94. https://doi.org/10.7202/602446ar 


\title{
Entre représentations hiérarchiques et pratiques complexes: les paradoxes de la décentralisation française
}

\author{
Pascal Roggero \\ MCF de sociologie \\ Responsable du CIRESS-LEREPS \\ Université de Toulouse 1
}

Omniprésent et apparemment irrésistible, le processus de mondialisation affecte l'ensemble des sociétés humaines. Si toutes sont travaillées à des titres et des degrés divers, peu, dans le monde occidental, apparaissent aussi profondément interrogées ${ }^{1}$ sur leurs fondements comme l'est la société française. Aimant à se penser dans une superbe singularité et encore convaincue de porter les idéaux messianiques de la Révolution, la plupart des élites de ce pays redoutent la fin de l'« exception » française.

À bien des égards, et en dépit de la crâne position internationale de son président, on tend à penser que cette société est saisie par le doute et, de fait, elle s'interroge, de manière assez obsessionnelle, sur son déclin (voir par exemple Baverez, 2003). L'histoire nationale récente résonne des manifestations d'une appréhension populaire des effets dévastateurs, ou redoutés tels, de la mondialisation. Mais si la société française semble plus pénalisée que beaucoup d'autres nations par le processus en cours, n'est-ce pas parce qu'elle est aussi atteinte dans ses fondements culturels et politiques?

\footnotetext{
${ }^{1}$ Signalons ici le travail de Simon Laflamme (2000) concernant la société canadienne, et son ambitieux projet de recherche sur «Les effets comparés de la mondialisation en Europe et en Amérique du Nord » auquel nous collaborons.
} 
Habituée à penser le monde sur un mode abstrait à l'image des Lumières qui décidèrent « d'assigner la société au tribunal de la Raison» (Cassirer, 1970: 70) età agir, hiérarchiquement et rationnellement, par l'entremise de l'État qui «transmet la loi et les ordres du gouvemement jusqu'aux demières ramifications de l'ordre social avec la rapidité du fluide électrique $»^{2}$, la société française, confrontée aux effets de la mondialisation, s'interroge sur la viabilité et la pérennité de ses institutions. Ayant incarné et largement exporté, depuis le Premier Empire, un modèle centralisé d'administration locale, l'État français a commencé à le remettre en question en 1982 en transférant aux collectivités territoriales - régions, départements et communes - certains pouvoirs, des compétences, les financements et les personnels associés. Cette politique de décentralisation est au cœur de l'action du gouvernement Raffarin depuis 2002.

Si l'histoire, l'analyse juridique et politique de la décentralisation ne sont plus à faire ${ }^{3}$, il en est une dimension qui demeure encore trop méconnue: il s'agit des représentations qui lui sont associées. Nous faisons l'hypothèse que les profondes réticences à la mise en œuvre de la décentralisation en 1982 de même que l'indifférence sceptique de l'opinion à l'endroit de l'action décentralisatrice du gouvernement Raffarin ne se comprennent vraiment qu'en explorant les représentations qui fondent les relations entre l'État et les collectivités territoriales. Autrement dit, il nous apparaît que la manière dont, à chaque époque, les principaux acteurs des relations entre l'État et les collectivités territoriales connaissent et comprennent ces demières en les reconstruisant dans leur système cognitif, pour ensuite les évaluer en les intégrant à leur système de valeurs, mérite un examen attentif. Ainsi, proposons-nous de remonter en amont des caractérisations politico-juridiques habituellement utilisées pour décrire les relations entre l'État et les collectivités territoriales, telles que leurs statuts par exemple, pour en rechercher les fondements cognitifs et idéologiques. C'est une perspective de réflexion transdisciplinaire - traversant l'histoire des institutions et des idées, le droit public et la sociologie notamment - qui rejoint en bien des points celle initiée par Rosanvallon depuis 1992 et qu'il décrit comme une « histoire philosophique du politique. » (1992, 1998, 2000: 34).

${ }^{2}$ De J.-A. Chaptal, ministre de l'Intérieur du Consulat, cité par J. Tulard (1985: 196).

${ }^{3}$ La dernière livraison, après bien d'autres, étant celle de J.-M. Ohnet (1996) à laquelle nous pouvons ajouter le beau livre de Deyon (1996). 
Il y a un double paradoxe dans l'histoire des relations que les collectivités territoriales françaises entretiennent avec l'État. Il est effectivement paradoxal de voir se succéder les régimes politiques et les textes constitutionnels depuis la Révolution alors même que les institutions locales connaissent, dans le même temps, une grande continuité. De même, lorsque les problèmes d'adaptation des institutions locales s'aggravent, nombre d'acteurs locaux et nationaux préconisent la décentralisation. Dans les années 1970, l'adhésion semble forte à cette perspective; pourtant il faudra attendre le coup de force politico-administratif d'un homme, Gaston Defferre, ministre de l'Intérieur et de la Décentralisation et maire de Marseille, pour que la décentralisation entre dans le droit positif en 1982. Le même décalage entre les aspirations présumées des acteurs et leur concrétisation décentralisatrice se retrouve durant les années 1990. En effet, si la quasi-unanimité se fait parmi les acteurs de la vie territoriale pour reconnaitre l'irréversibilité du processus engagé en 1982 et s'en féliciter, elle existe aussi pour souhaiter la deuxième vague de la décentralisation qui a tardé. Il fallut attendre l'action du gouvemement Raffarin à partir de 2002 pour qu'elle soit mise en œuvre et, aujourd'hui encore, discutée au Parlement avec, là encore, une réserve qui prend la forme de l'indifférence de la population et de l'hostilité d'une partie des élites administratives. Dans leur stabilité comme dans leur changement, les relations entre l'État et les collectivités territoriales recèlent donc un paradoxe. Stables alors que tout change, elles s'adaptent difficilement alors que leur changement est très généralement souhaité. Avant de chercher à expliquer cette situation paradoxale $e^{4}$ disons un mot de la méthode ici employée.

Par delà les circonstances historiques, les rapports de pouvoir et de classe, par-delà les cohérences purement juridiques, l'action de l'État à l'égard des collectivités territoriales est aussi tributaire des principes idéologiques et des schèmes cognitifs par lesquels les élites politiques appréhendent, comprennent et expliquent le monde qui les environne. Mais comment repérer ces principes idéologiques et ces schèmes cognitifs? C'est par l'interprétation des textes politico-juridiques - qu'il s'agisse de l'ensemble des textes juridiques et de leurs travaux préparatoires; des discours, des écrits et des joumaux politiques ou bien encore des informations circulant dans l'appareil d'État comme les rapports préfectoraux par exemple - que nous tenterons de les identifier. À l'instar de Panofsky (1967) qui mit en lumière l'existence d'une influence de la pensée scolastique à laquelle les architectes des cathédrales gothiques avaient été formés, sur les plans de ces dernières, nous recherche-

\footnotetext{
${ }^{4}$ La recherche que Rosanvallon mène depuis deux ans au Collège de France s'inscrit dans cette perspective.
} 
rons une " homologie structurale" (Berthelot, 1990) entre, d'une part, les relations entre l'État et les collectivités territoriales et, d'autre part, les représentations qui leur sont associées. Ainsi, par exemple, la tutelle par laquelle les actes des collectivités territoriales ont fait longtemps l'objet d'un contrôle d'opportunité par le représentant de l'État est à mettre en rapport, sur le plan idéologique, avec la supériorité politique de la nation sur la commune et, sur le plan cognitif, avec la conception d'un monde ordonné ainsi qu'en témoigne la métaphore physicaliste de Chaptal citée plus haut. À des relations hiérarchiques entre l'État et les collectivités territoriales correspond donc une représentation elle-même de nature hiérarchique. Les représentations scientifiques exercent une influence importante sur les représentations en général et donc aussi sur celle des relations entre l'État et les collectivités territoriales. À cet égard, le concept de « contrat social épistémologique $»^{5}$ développé par Le Moigne nous semble tout à fait pertinent pour identifier la nature des représentations scientifiques et leurs répercussions sociales. Nous l'utiliserons notamment pour décrire la composante cognitive de la représentation des relations entre l'État et les communes.

Partant de ce type d'approche, nous faisons l'hypothèse que cette situation paradoxale de la décentralisation en France trouve une part de ses origines dans l'économie cognitive et les principes idéologiques qui ont fondé le cadre politico-juridique des relations entre l'État français et les collectivités territoriales depuis deux siècles. Plus précisément, des représentations que nous pouvons qualifier de hiérarchiques, principalement issues des Lumières via la Révolution et l'Empire, ont contribué à inspirer et légitimer un cadre juridique centralisateur biséculaire. La forte légitimité et la cohérence de ce cadre ont interdit toute véritable adaptation politico-administrative jusqu'au début des années 1980, cette dernière s'effectuant "à la hussarde " en bousculant l'habitus centralisateur. Si les relations concrètes entre l'État et les collectivités territoriales ont évolué, de fait, vers plus d'autonomie, les représentations hiérarchiques n'ont pas, pour autant, disparu. Leur vigueur, encore actuelle, constitue un élément permettant de comprendre pourquoi

\footnotetext{
${ }^{5}$ Qui détermine non seulement ce qu'est la scientificité mais aussi la place de la science et donc des scientifiques dans la société (Le Moigne, 1995).

'Ainsi que l'a expliqué R. Quilliot, ministre du Logement en 1981 et proche de G. Defferre au moment de la rédaction de la grande loi de décentralisation, la loi du 2 mars 1982 relative aux droit et libertés des communes, départements et régions, qui, pour la première fois en France, entame la logique centralisatrice des relations entre l'État et les collectivités territoriales.
} 
«la seconde vague » de la décentralisation, initiée par le gouvernement de Raffarin en 2003, s'effectue au mieux dans l'indifférence empreinte de scepticisme, au pire dans l'hostilité déclarée. Nous tenterons de montrer que, pour que la décentralisation soit assumée et revendiquée par l'ensemble des acteurs territoriaux, une évolution en profondeur de la représentation des relations État-collectivités territoriales est nécessaire dans le sens d'une reconnaissance de leur complexité. Dans notre traitement de cette large question des relations entre l'État et les collectivités territoriales, nous nous limiterons au cas de la commune et particulièrement au statut juridique que l'État lui reconnait.

Voyons d'abord quelle est la cohérence idéologique et cognitive de la représentation qui fonde les relations entre l'État et les collectivités territoriales en général et, plus précisément, les communes depuis l'époque révolutionnaire et napoléonienne avant d'examiner en quoi cette représentation doit changer pour assurer la pérennité de la décentralisation en France aujourd'hui.

\section{Les fondements idéologiques et cognitifs du modèle hiérarchique des relations entre l'État et les communes: le cas de l'unité statutaire des communes}

Le temps historique n'est pas linéaire. Il existe, dans l'histoire des sociétés et des institutions, des périodes critiques durant lesquelles semble se décider le sort des collectivités. Avec les guerres et les dépressions économiques, les révolutions politiques constituent les formes les plus communes de la crise historique. La Révolution française qui marque l'entrée de la France dans la modernité politique en donne la plus fameuse illustration. Ce processus révolutionnaire se traduit par un changement institutionnel de grande ampleur qui jette les bases des régimes ultérieurs. La commune, qui n'échappe pas à ce mouvement, entre alors en Révolution.

Dans ce jugement en raison des institutions promues par la Révolution, l'esprit des Lumières s'exprime fortement. Dans le domaine de l'administration locale, comme dans bien d'autres, il commande de reconstruire pour sortir de l'emprise de la tradition. Dans cette reconstruction, la commune doit être politiquement subordonnée et juridiquement unifiée. En effet, la Révolution érige la nation comme seul espace politique légitime dans lequel se déploie la citoyenneté et, d'où, comme l'entendait l'abbé Sieyès, émanait la souveraineté. Dès lors, et même s'il faut faire la différence entre les hommes de 1789 et leurs successeurs de 1791 et surtout de 1793 et de l'an VIII, la commune apparait comme une institution infrapolitique, une sorte de "sousproduit » de la nation à laquelle elle doit être strictement subordonnée. Les 
choix collectifs nationaux doivent s'imposer au nom d'une cohérence idéologique où les principes révolutionnaires essentiels tels que la prépondérance législative, l'unité nationale ou l'égalité de droits se renforcent les uns les autres.

Face à l'extrême diversité des institutions territoriales de l'Ancien Régime, marquée par les privilèges, les révolutionnaires entendent créer un statut communal unifié. Ici s'expriment autant une orientation idéologique pour l'égalité qu'une inclination cognitive pour l'uniformité issue des représentations scientifiques de l'époque, ce que Rosanvallon qualifie dans sa leçon inaugurale au collège de France de «culture politique de la généralité» (2002). C'est donc une nouvelle vision du monde, celle d'un monde que la raison permet de connaître et de transformer, qui fonde l'œuvre institutionnelle de la Révolution. Rarement, et peut-être jamais, dans l'histoire de France, des institutions nouvelles ont autant dû à des représentations. Cette empreinte des représentations révolutionnaires perdure encore de nos jours. Nous nous attacherons donc à rechercher les représentations tant idéologiques que cognitives qui, à partir de la Révolution, fondent les relations de l'Etat avec les communes. Comme nous le verrons, les représentations scientifiques ont leur part dans cette influence.

\subsection{La cohérence idéologique de la représentation associée à l'unité statutaire des communes}

Les principes que les Lumières avaient posés comme fondamentaux et qui structurent encore notre droit public - les principes de légalité, d'unité, d'égalité et de centralité - expriment le mieux le nouvel esprit public qui a pu inspirer les institutions locales créées lors de la Révolution.

Après avoir abordé les raisons de la création institutionnelle de la Révolution, nous tenterons de montrer quels ont été les principes qui en ont ordonné la mise en œuvre.

\subsubsection{Les raisons d'un changement: la commune dans la toumente institutionnelle}

Nous le savons, la Révolution, s'attaquant à l'ordre politique, visait aussi l'ordre social. Les ordres abolis, il fallait que les institutions locales qui leur étaient liées le fussent aussi. Mais, à cette destruction, les révolutionnaires entendaient ajouter une construction, celle d'un nouvel ordre sociopolitique

\footnotetext{
7 Qui inspira à Sieyès, «obsédé par l'unité nationale », le terme d' «adunation » pour signifier la tension de la nation vers l'unité (Bredin, 1988: 167-175).
} 
articulé sur les notions de nation et de représentation. Il s'agissait de bâtir ce nouvel édifice sur les décombres de l'ancien.

$\grave{A}$ une stratification sociale encore largement fondée sur la naissance, les révolutionnaires voulurent substituer une distribution des positions sociales selon le mérite. Ce critère méritocratique devait s'appliquer aussi aux fonctions politiques dont la désignation devait se faire entre les hommes "sans autre distinction que celle de leurs vertus et de leurs talents ". Dès lors, la suppression des ordres induit celle de la division territoriale qui « les rappelant sans cesse peut offrir la tentation et les moyens de les rétablir» ( $L e$ Moniteur universel, $n^{\circ}$ 79: 321). À travers cet épisode, nous mesurons d'ailleurs que les institutions locales ont toujours à voir avec la hiérarchie sociale. L'égalité juridique posée par la déclaration des droits du 26 août 1789 constitue donc un facteur essentiel de ce changement local. Mais à l'égalité s'ajoute l'efficacité pour justifier la nécessité du changement.

Dans un espace unifié aux circonscriptions uniformes, la gestion des affaires publiques était présentée comme s'exerçant à la fois plus près des citoyens auxquels nous conférions le pouvoir d'élire leurs dirigeants et de manière plus conforme à la raison. Cette affirmation de l'égalité politique et de l'efficacité administrative ne pouvait se faire dans le cadre des vieilles communautés. En effet, fonder le nouveau système d'organisation administrative sur les vieilles structures communales aurait représenté une double erreur. De par leur faiblesse, ces institutions étaient, dans les campagnes, «à la merci des seigneurs, des curés ou de quelques notables » et, dans les villes, «dominées par le crédit des principaux citoyens ». En conséquence « l'unique moyen pour émanciper l'autorité communale est de la distribuer en plus grandes masses » (Le Monieur universel, $\mathrm{n}^{\circ}$ 65: 266). Cette exigence politique correspondait à un impératif administratif dans la mesure où, «plus puissants » et « moins nombreux », ces nouveaux corps politiques « pourraient devenir utiles sous une infinité de rapports publics » (ibid.) à la différence des vieilles communautés, de taille trop réduite. Nous pouvons ainsi constater que les débats contemporains sur l'optimum de taille des communes sont loin d'être nouveaux.

Si la Constituante n'a pas supprimé la commune comme l'y engageait le comité, elle a tout de même inscrit son action dans l'orientation idéologique définie par ce dernier. Cet édifice institutionnel local s'inscrit dans l'élaboration d'un nouvel ordre national et représentatif.

À cet ensemble fragmenté, constitué de multiples privilèges attachés tant aux personnes qu'aux territoires, que constitue le royaume, la Révolution a

${ }^{8}$ Article 6 de la Déclaration des droits de l'homme et du citoyen du 26 août 1789. 
substitué un espace abstrait où la loi s'applique uniformément et où la citoyenneté et l'égalité des droits s'affirment: la nation'. Cette entité politique nationale s'impose à toutes les autres; elle est souveraine. Cette souveraineté, jusque-là réservée au seul monarque, est ainsi transférée, par le processus révolutionnaire, à la nation. Cette souveraineté s'exprime par la loi qui est faite par les représentants des citoyens réunis en " corps législatif ». Cette représentation constitue le deuxième grand fondement du nouvel ordre politique.

En effet, la nouvelle division territoriale a aussi, et peut-être surtout, pour objet de constituer la base d'un système permettant « d'établir une représentation exactement proportionnelle entre toutes les parties du royaume » (Thouret, 29 septembrc 1789; Le Moniteur universel, $\mathrm{n}^{\circ}$ 74: 265) lors de l'élection des députés. Cette égalité de représentation s'accompagne d'une unité de représentation puisque « les représentants nommés à l'assemblée nationale ne pourraient être regardés comme les représentants d'un département particulier mais de tous les départements, c'est-à-dire de la Nation entière » (Le Moniteur universel, $\mathrm{n}^{\circ}$ 79: 321).

Ce nouvel ordre politique, dont dépendent profondément le statut communal et son caractère uniforme, passe par la loi.

\subsubsection{L'uniformité statutaire des communes et le principe de légalité}

Avec la Révolution, la conception rousseauiste de la loi va s'imposer et induire l'uniformité statutaire des communes.

Le principe de légalité signifie ici que seuls les députés sont autorisés à élaborer la loi qui devient, à la faveur de la Révolution et à la différence de l'Ancien Régime, la principale source du droit. En effet, la monarchie absolue était caractérisée par le pouvoir arbitraire et la domination du monarque dont les droits inaliénables de l'homme et de la raison exigeaient la suppression. Comme la raison, à laquelle les hommes ont naturellement accès, il existe, selon les Lumières, "des droits intangibles attachés à la personne humaine » (Cassirer, 1970: 247). Leur respect nécessite la loi car, pour sortir de l'arbitraire, les hommes doivent se soumettre sans exception à des lois impersonnelles devant lesquelles ils sont tous égaux. La loi permet aussi de concilier l'idéal de l'unité de l'État et l'aspiration à l'autonomie et l'égalité des individus.

La loi, selon Rousseau, représente " la plus sublime des institutions humaines ", notamment parce qu'elle seule permet de concilier la liberté

\footnotetext{
9 Indéniablement pensée dans l'abstraction par Sieyès, la nation aurait, selon certains, une origine lointaine liée aux communautés de base (Bourjol, 1990).
} 
individuelle et l'exercice de la nécessaire autorité en collectivité. Elle rappelle d'abord qu'il n'est de pouvoir que délégué. Mais, surtout, par le « contrat social » dont elle procède, elle exprime la volonté générale, celle de tous et de chacun à la fois. De la sorte, en acceptant la loi, l'individu ayant conclu un pacte avec tous les autres n'obéit pourtant qu'à lui même ${ }^{10}$. L'individu, naturellement libre, peut, dans ces conditions, accepter le pouvoir qui n'est légitime que parce qu'il est subordonné à la loi. La loi, expression de la volonté générale, possède la souveraineté. C'est dans le cadre national que la Révolution entendra mettre en œuvre ce principe de légalité. Le rapport du comité de constitution s'inquiéta d'ailleurs d'un possible déclin de « l'exactitude et de l'uniformité dans le régime exécutif » qui aurait pu faire « dégénérer la Nation en démocratie dans l'intérieur des provinces (Thouret, 29 septembre 1789; Le Moniteur universel, $\left.n^{\circ} 74: 265\right)$. Ce principe contribue à l'uniformité statutaire des communes.

Cette loi qui ne « connait pas d'autorité supérieure " " "doit être la même pour tous ${ }^{12}$. Si ce penchant unificateur et égalitariste de la loi révolutionnaire s'explique dans le court terme par une réaction à l'Ancien Régime, il a fondé le principe d' « identité institutionnelle " (Favoreu, 1983: 19) qui a été appliqué aux collectivités territoriales jusqu’à très récemment encore. En finir avec les privilèges territoriaux semblait imposer fort logiquement l'uniformité statutaire des nouvelles collectivités créées. Il reste que ce prisme uniformisant a, bien après sa mise en œuvre, durablement figé la forme institutionnelle des collectivités territoriales. Mais il est loin d'en être le seul facteur explicatif. Le principe d'unité est à ranger dans cette catégorie.

\subsubsection{De l'unité nationale à l'uniformité statutaire des communes}

Si la quête de l'unité fut une préoccupation constante de la monarchie, la Révolution en a fait un objectif majeur. Ce souci unitaire imprégna le rapport Thouret qui mit au premier plan l'unité de la nation fondée sur la triple unité

\footnotetext{
1" "Chacun se donnant à tous ne se donne à personne; et comme il n'y a pas un associé sur lequel on acquiert le même droit qu'on lui cède sur soi, on gagne l'équivalent de tout ce qu'on perd et plus de force pour conserver ce qu'on a ». J. J. Rousseau, Le Contrat social, livre I, chap. 6. Nous verrons plus loin qu'il s'agit d'un obstacle sur lequel a buté la pensée politique.

${ }^{11}$ Article 2 de la Section I du Chapitre I du Titre III de la constitution du 3 septembre 1791.

${ }^{12}$ Article 6 de la Déclaration des droits de l'homme et du citoyen de 1789.
} 
de la représentation, de l'espace et des modalités d'application de la loi. Cette unité nationale justifiait aussi l'uniformité statutaire des communes.

Cette unité fut conçue de manière abstraite à travers cette entité nouvelle qu'était la nation. Cet espace politique neuf, à la fois égalitaire et rationnel, se substitua à l'espace traditionnel du royaume marqué par les privilèges et construit par l'histoire. Unifier le pays passait par la suppression des anciennes administrations (Décret du 22 décembre 1989) provinciales et locales, vecteurs de particularismes rétrogrades, et leur remplacement par de nouvelles institutions tant locales que nationales. Le programme était donc clair; il s'agissait de " créer et organiser en entier " « un nouveau corps politique et élever sur une base commune le double édifice de la représentation nationale et de l'administration provinciale et communale » (Le Moniteur universel, $\mathrm{n}^{\circ} 79: 321$ ). Cette édification passait parl'uniformité qui imposait une même organisation municipale à toutes les communes. Nous savons qu'une inspiration similaire présida à la création des départements (OuzoufMarignier, 1989), même si les frontières provinciales furent aussi prises en compte pour le découpage des quatre-vingts circonscriptions créées. Cette uniformisation fut l'objet des critiques virulentes des contre-révolutionnaires qui, de Burke (1912) à Maistre (1988) et Maurras (1948), stigmatisèrent l'abstraction, l'impersonnalité et l'uniformité des institutions. Ces traits, présents dans l'institution communale instaurée par la Révolution, préexistaient dans la conception du monde dominante chez les penseurs des Lumières.

Très vite, l'unité du royaume s'est accompagnée de son indivisibilité. Cette association unité-indivisibilité a ensuite été appliquée à la République par la constitution montagnarde de 1793 et demeure encore un principe fondamental du droit des collectivités locales. Elle a constitué une référence centrale pour tous les défenseurs sourcilleux du centralisme qui se sont toujours opposés en son nom à l'émancipation des communes et à la diversification de leur statut. De l'unité nationale à l'uniformité statutaire des communes, la conjonction historique apparait donc clairement.

En partant des dispositions juridiques, nous avons tenté d'établir, par la recherche de relations de congruence, la nature des fondements politicoidéologiques de l'uniformité statutaire des communes. Cette recherche est évidemment fondée sur l'hypothèse, généralement admise, que l'action des hommes dépend, en partie au moins, de leurs représentations. En effet, ce que nous appelons les « fondements politico-idéologiques » de l'uniformité statutaire des communes correspond à une partie de la représentation qui oriente les relations entre l'État et les communes. 
Figure 1

La cohérence « jacobine » de la représentation des relations État-communes

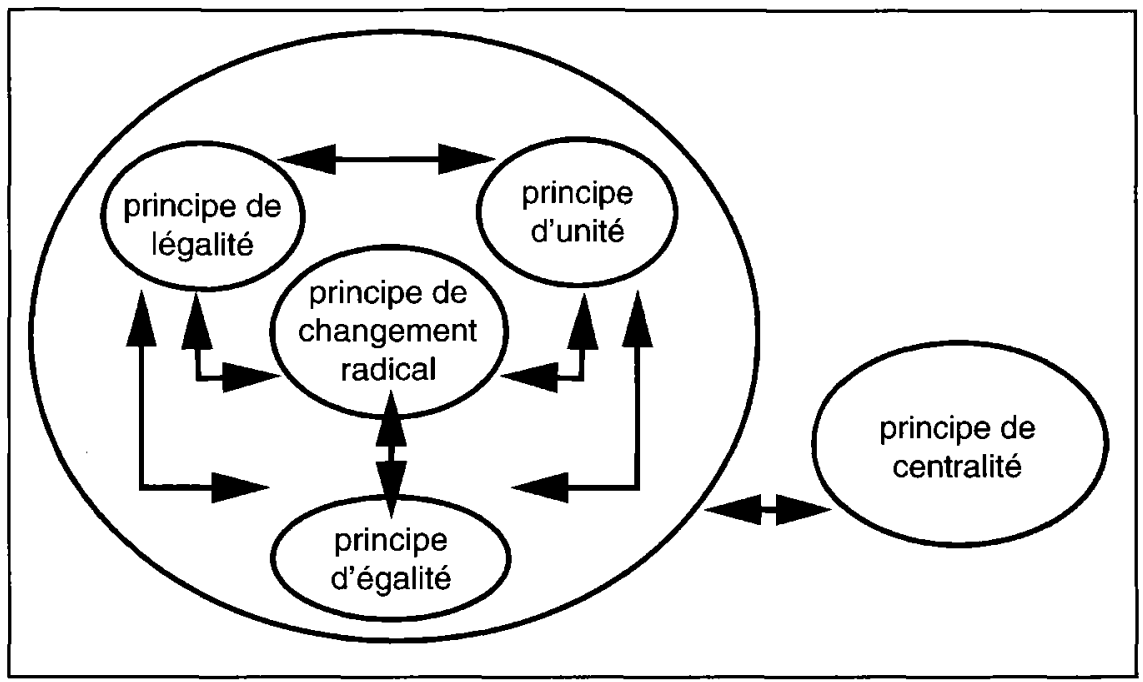

À travers le seul trait de l'uniformité statutaire, nous voyons se dessiner une représentation très cohérente au niveau politique et idéologique. Le statut des collectivités locales devant être changé profondément, le principe de changement occupe donc une place centrale. Ce changement passe par la loi, norme impersonnelle et générale qui exprime la souveraineté de cette entité abstraite qu'est la nation. Cette loi définit un intérêt général transcendant rousseauisme oblige - et s'applique, de manière égale, dans un espace unitaire: la nation. Il se produit un effet de renforcement de ces principes les uns après les autres. Une telle dynamique débouche sur une représentation que nous pouvons qualifier de hiérarchique dans la mesure où elle subordonne la commune à l'État.

\subsection{Au nom des Lumières de la raison ou les fondements cognitifs de l'uniformité statutaire des communes}

Qu'il s'agisse des principes de changement, de légalité, d'unité ou d'égalité, ils trouvent tous leurs fondements cognitifs dans la conception du monde des Lumières. Examinons cette filiation cognitive qui place la raison au cœur de l'ensemble. 


\subsubsection{La raison commande... le changement}

Entendant rompre avec la tradition et sa légitimation religieuse, les révolutionnaires doivent proposer une nouvelle weltanschauung. Ils la trouvent dans la représentation d'un monde gouverné par une raison immanente ainsi que le montrent les sciences physiques de l'époque. Ils peuvent dès lors traduire la société entière au "tribunal de la raison » et envisager de reconstruire rationnellement le monde sociopolitique.

Le projet politique de changement s'inscrit donc dans un bouleversement des représentations opéré par la philosophie des Lumières au XVIII siècle. Ce siècle est traversé par une énergie, une force par laquelle les hommes croient accéder à la connaissance du monde et d'eux-mêmes: la raison. Cette raison n'est plus celle des "vérités éternelles " du XVII ${ }^{e}$ siècle qui devait s'articuler à la raison divine. Non, elle s'est émancipée de cette dépendance et entend éclairer le monde sans se soumettre à un a priori transcendantal. Cette émancipation, essentielle sur le fond, résulte en partie d'un changement de démarche dans la science physique.

Avec Descartes, Malebranche, Spinoza ou Leibniz, le raisonnement était dominé par la déduction; il s'agissait de partir de principes, d'axiomes ou de définitions abstraits pour atteindre, par des démonstrations en chaîne, l'explication des faits particuliers. D'une cause première découlait rigoureusement, c'est-à-dire par la médiation de la démonstration, la chaîne du connaissable. Cette démarche scientifique est formellement proche de la tendance philosophique à construire des « systèmes $~^{1.3}$ à partir de «l'idée première d'un être suprême ».

Avec Newton, le monde des phénomènes s'impose. Il faut les saisir par l'expérience et l'observation et, seulement ensuite, rechercher la forme mathématique qu'ils recèlent pour enfin accéder à la loi explicative générale. Newton, s'il recourt aussi, bien sûr, à la déduction, entend poser la démarche inductive comme nécessaire à toute production scientifique. Dès lors, c'est dans le monde qui se déroule sous nos yeux qu'il faut chercher les manifestations concrètes de la raison et non dans une vérité révélée. Loin d'exclure nécessairement la théologie et la religion, la physique du XVIII ${ }^{\mathrm{e}}$ siècle et la conception du monde qu'elle véhicule se construisent néanmoins contre elles.

\footnotetext{
${ }^{13}$ Ce qui amènera Condillac dans Le traité des systèmes à distinguer «l'esprit de système » qui bâtit à partir d'un dogme et "l'esprit systématique » qui recherche systématiquement à relier le «monde positif» et le «monde rationnel » (Cassirer, 1970: 42).
} 
Le XVIII ${ }^{\mathrm{e}}$ siècle rompt ainsi avec la conception en quelque sorte patrimoniale de la raison du siècle précédent dans laquelle nous puisons la vérité comme dans un trésor. Il s'agit, au contraire, de la concevoir comme une énergie qui conduit à découvrir la vérité dans une sorte de quête permanente de l'explication scientifique qu'il faut appliquer aussi à la société.

Cassirer a écrit que la raison a «le pouvoir de lier et de délier» (1970: 48). Elle lie les phénomènes dans les formes mais, surtout, elle délie l'esprit des " vérités toutes faites » issues de l'autorité, de la tradition ou de la religion. De ce fait, « la société est assignée au tribunal de la raison, interrogée sur la légitimité de ses titres, sur les fondements de sa vérité et de sa validité. " (op. cit: :53). C'est donc devant ce tribunal que les révolutionnaires traduisent les institutions d'Ancien Régime, parmi lesquelles les institutions locales. Le verdict est sans appel: il faut détruire pour reconstruire, non pas ré-former mais re-former.

En effet, ces institutions constituent un ensemble hétéroclite, inégalitaire, et décadent - «les débris incohérents de l'ancienne monarchie » (Le Moniteur universel, $n^{\circ}$ 79: 321) - dont la corruption la plus importante consiste à n'avoir pas été élaborées en vertu de la raison. L'empire que la raison exerce sur les hommes ne peut souffrir l'existence de tels reliquats, qui sont autant d'entraves. Il faut porter sur ces institutions « au plus haut degré les lumières et l'esprit public, c'est-à-dire la véritable force et la véritable puissance » (ibid.). Détruire, la raison le commande; construire, la raison l'ordonne.

En effet, les hommes partageant tous l'exercice de la raison, abattre des institutions imposées par la tradition et l'autorité constitue une restauration légitime de l'ordre rationnel du monde. À cette restauration rationnelle, ni la société ni l'État ne sauraient échapper et la reconstruction doit s'opérer dans la transparence.

De Hobbes à Rousseau, n'avons-nous pas tenté de concevoir une explication rationnelle de la genèse de l'État? La méthode employée consistait à considérer l'État, mais aussi les organes locaux, comme des « corps » qu'il fallait recomposer à partir des volontés particulières. Ces dernières, unités de base des « corps » politiques, s'accordaient dans le cadre d'un contrat pour constituer l'État. Dans ce procès de décomposition-recomposition, la démarche analytique, propre aux sciences de la nature, est appliquée au monde social.

Exigence éthique en même temps que nécessité rationnelle, la destructionreconstruction des institutions s'impose et nécessite la loi. 


\subsubsection{La raison et la loi ou la légalité rationnelle du monde}

La légalité juridico-politique que le nouvel ordre instaure et applique au statut communal n'est-elle pas la "traduction ${ }^{14}$ de la légalité du monde physique ${ }^{15}$ ?

Si Newton, à la différence des physiciens du XVII siècle, réhabilite le fait et la démarche inductive, c'est parce qu'il postule l'existence, dans le monde empirique, de formes mathématiquement saisissables, véritables expressions de lois générales. Il présuppose donc «l'ordre et la légalité parfaite » (Cassirer, 1970: 43) du monde des phénomènes. La soumission du réel - dans sa totalité et dans sa diversité - à des règles générales établies par la démarche scientifique, voilà l'ambition nomothétique de la raison. D'ailleurs, Montesquieu ne nourrit-il pas un même projet nomothétique quand, dans L'Esprit des Lois, il se propose d'étudier « les rapports nécessaires qui dérivent de la nature des choses » dans le domaine des régimes politiques principalement?

La loi est investie par les révolutionnaires d'une mission rationalisatrice. Dans l'incohérence du vieux monde, la tâche du législateur concevant une nouvelle organisation administrative est " difficile ", " effrayante par la nouveauté et la hardiesse du plan, l'immensité des détails et une foule de difficultés physiques et morales. » (Le Moniteur universel, $n^{\circ}$ 79: 321). Seul «le génie de la liberté ", c'est-à-dire la raison, " pouvait ne pas les trouver invincibles » (ibid.). De cette quête difficultueuse mais inéluctable de la raison parmi les linéaments du monde, la loi doit être le produit. Peu de lois ont autant que les décrets des 14 et 22 décembre 1789 représenté cette tentative de reconstruction rationnelle du monde.

De la même manière que la loi physique englobe à la fois le singulier et l'universel, la loi politique s'applique à chacun de manière impersonnelle. Comme l'attraction universelle, elle tend vers l'unité.

\subsubsection{L'unité et la raison ou l'unité rationnelle du monde}

La construction abstraite de l'unité nationale par Sieyès et les révolutionnaires rappelle, en bien des points, l'unité du monde physique que Newton établit avec sa loi de l'attraction universelle.

\footnotetext{
${ }^{14}$ Au sens où l'entend Latour (1989).

${ }^{15}$ Hypothèse envisagée par Dilthey (1942), par Groethuyssen (1956) et par Ruelle (1993: 441-471).
} 
Quand, remaniant les puissantes contributions théoriques de Kepler et de Galilée et inventant le calcul infinitésimal, Newton parvient à établir sa fameuse loi de l'attraction universelle, il consacre le triomphe de l'esprit analytique moderne. Par cette formule « cosmologique ", " il soumet la totalité du réel à une règle unique » (Cassirer, 1970: 44). Tous les phénomènes sont ainsi « unifiés » sous l'empire de cette loi universelle.

L'impact socioculturel de cette découverte est immense. Relayée de toute part, notamment par les auteurs de L'Encyclopédie, elle influence considérablement la structure cognitive que les hommes vont appliquer au monde, y compris au monde social.

Cette conception unitaire habite les textes révolutionnaires. Ainsi, tant le régime représentatif que l'organisation territoriale témoignent de cette construction unitaire qui dans " un mouvement uniforme fait arriver la représentation nationale au corps législatif " (Thouret, 29 septembre 1789; Le Moniteur universel, $\mathrm{n}^{\circ}$ 79: 255).

Tout comme la loi newtonienne de l'attraction gouverne l'univers, la loi politique gouverne la nation selon le principe d'égalité.

\subsubsection{La raison et l'égalité: une égalité abstraite}

Comme dans le monde physique, l'égalité politique passe par la légalité. En effet, en établissant, par la loi de l'attraction universelle, que l'ensemble du monde physique est soumis à une règle unique, Newton pose non seulement un principe d'unité mais aussi, d'une certaine manière, un principe d'égalité entre les objets le constituant. À l'échelle de la société, la loi d'inspiration rousseauiste, à la fois générale et impersonnelle, doit s'appliquer, avec la régularité de la loi physique, au monde politique qu'ainsi elle unifie et égalise. L'application d'une règle unique, de nature nécessairement abstraite, à des " réalités » concrètement très différentes, constitue l'un des fondements cognitifs de la conception du monde des Lumières. Nous savons qu'il a marqué durablement le statut des communes françaises. L'idée qu'un traitement égal de situations inégales pouvait susciter un surcroît d'inégalité ${ }^{16}$ était sans doute trop en contradiction avec l'orientation idéologique et la vision unitaire, voire universaliste, du monde qui prévalaient à l'époque et comptent encore aujourd'hui.

\footnotetext{
${ }^{16}$ Cette idée est présentée d'abord dans la critique marxiste de l'égalité formelle par les socialistes, notamment à partir de 1848 (Donzelot, 1984), et théorisée ensuite par Rawls (1987).
} 


\subsubsection{Essai de schématisation}

La logique cognitive de la représentation associée à l'uniformité statutaire des communes apparaît nettement à la lecture des textes et peut être succinctement caractérisée. L'utilisation permanente d'adjectifs renvoyant à une conception « mathématique » du monde par les textes révolutionnaires sur l'organisation territoriale apparait particulièrement significative. Ainsi est-il question de "proportionnalité », de " part égale et fixe ", de " part de députation », de « bases », de multiples nombres « sept cent vingt, quatrevingts, vingt-sept... ", de fractions correspondantes; bref, il s'agit de cette « division mathématique presque idéale » que critiqua Mirabeau.

Carte $n^{\circ} 1$ : Les circonscriptions administratives d'Ancien Régime

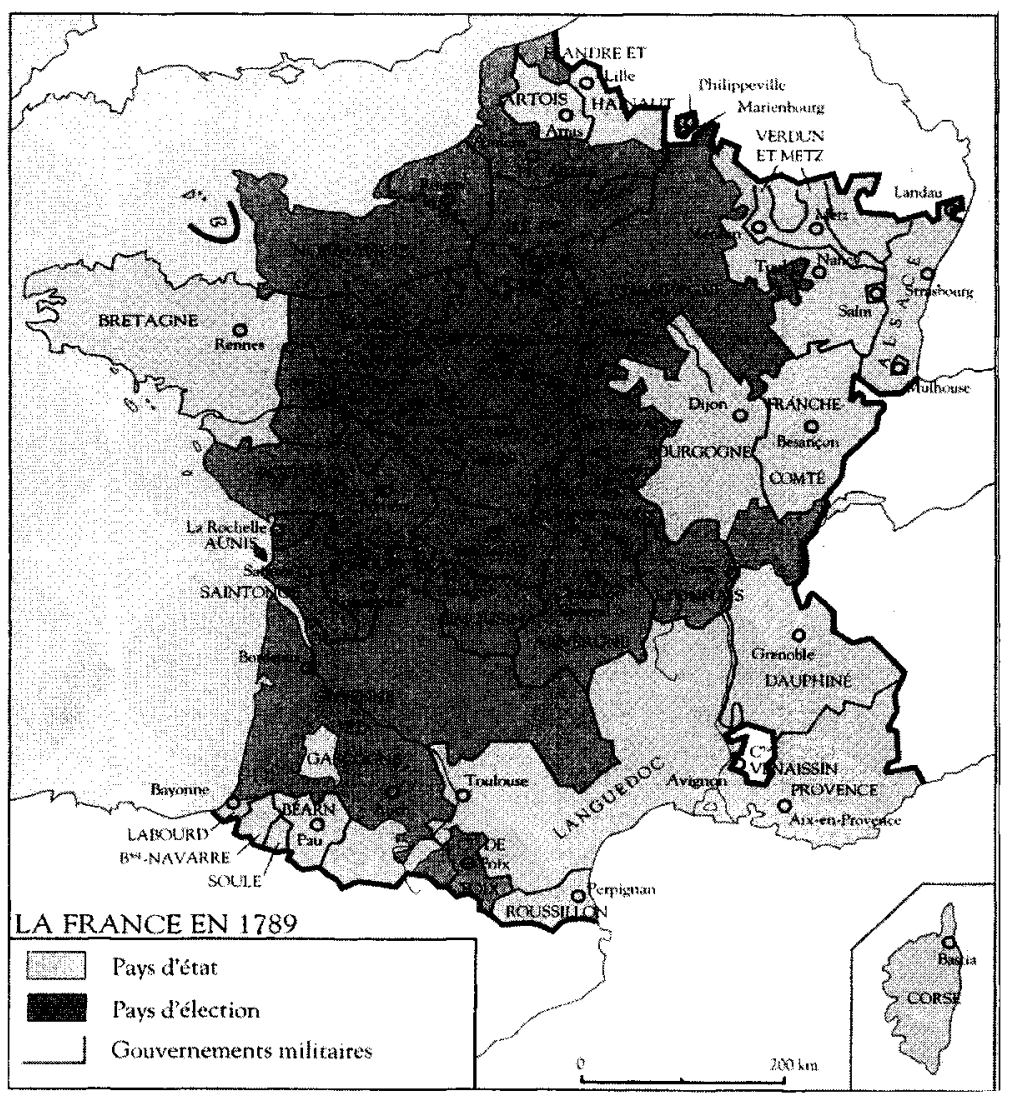


Carte $\mathrm{n}^{\circ}$ 2: Le projet de découpage « géométrique » du rapport de Thouret

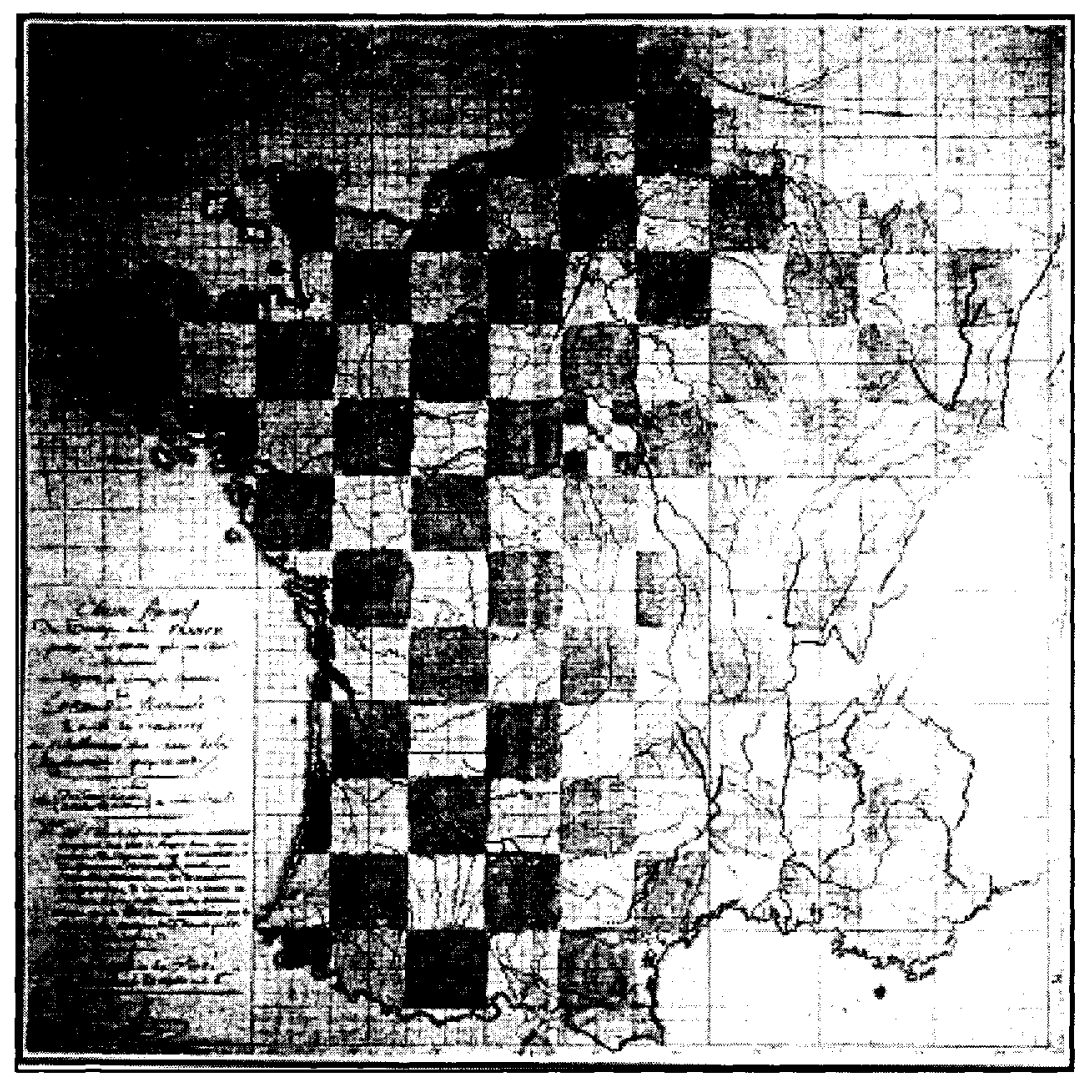

La carte des circonscriptions administratives que les hommes de 1789 entendent substituer aux " débris incohérents de l'ancienne monarchie » illustre bien cette volonté de "prescrire un ordre simple et immuable » et de «déterminer avec précision " ${ }^{17}$ l'ordre politique des choses. Nous en donnons une illustration avec les cartes 1 et 2 ci-dessus $^{18}$. Sur le modèle de la physique newtonienne se structure une représentation du monde politico-institutionnel.

${ }^{17}$ Gazette nationale..., n 05 et $\mathrm{n}^{\circ} 79$, op. cit. , 265-266 et 321-322.

${ }^{18}$ Deyon (1996: 108-109). 
Elle peut être caractérisée comme rationnelle sur le mode du XVIII siècle, déterministe selon Laplace ${ }^{19}$, voire mécaniste comme le montre la figure 2.

Figure 2

La nature cognitive de la représentation liée à l'uniformité statutaire

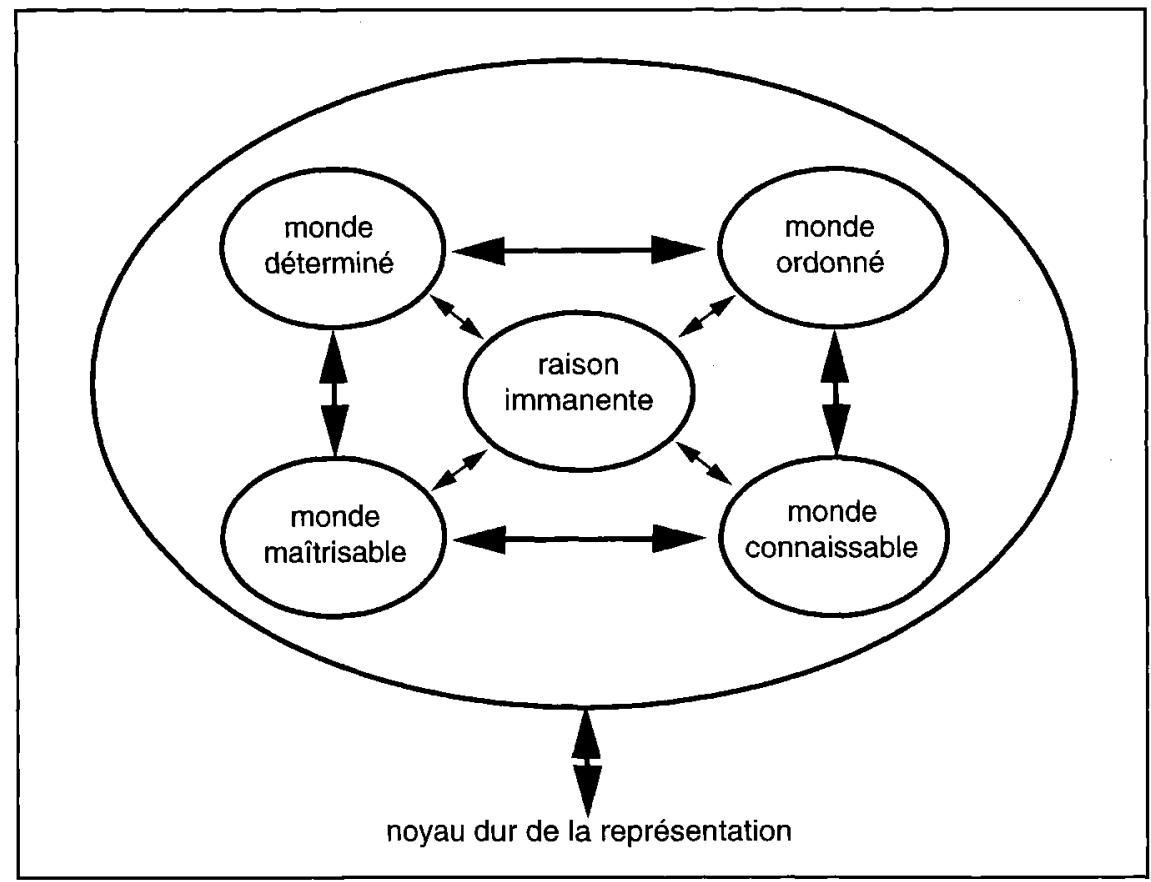

Garant d'une application uniforme de la loi sur le territoire, investi de l'autorité et de la défense des intérêts nationaux, et porteur, plus généralement, d'une « œuvre de civilisation ", l'État et son représentant local, le préfet, s'inscrivent dans un ensemble de représentations qui fondent leur rôle de

${ }^{19}$ Physicien d'envergure, Laplace a inventé, pour décrire l'ordre du monde, la figure métaphorique d'un "démon " auquel il a laissé son nom. E. Morin le décrit ainsi: «L'ordre souverain des lois de la nature est absolu et immuable. Le désordre en est exclu, de toujours, à jamais. Seule l'infirmité de notre entendement nous interdit de concevoir dans sa plénitude l'universel, impeccable, inaltérable, irrévocable déterminisme. Mais un démon, comme l'avait imaginé Laplace, capable d'observer tout l'univers à un instant donné et en connaissant les mois serait capable de recons- tituer tous les événements passés et d'en prédire les événements futurs » (1977:3). 
tuteur du territoire. La situation politique et sociale de la France du XIX siècle justifie pleinement cette fonction. La démocratie locale ne se décrète pas et l'épisode chaotique de 1789 à 1791 le démontre. Ainsi que l'a montré Agulhon, La démocratie au village (1979) est venue d'en haut, quand les enjeux locaux se sont progressivement émancipés des conflits familiaux et interpersonnels pour accéder à une structuration politique à l'image du débat national. Cette France rurale, « des terroirs » (Weber, 1983) doit encore faire, sur de larges parties de son territoire, l'apprentissage de la langue française et du sentiment national. L'école, la conscription, le chemin de fer et l'action préfectorale y contribueront fortement. Mais, il est loin le temps où un député pouvait s'adresser à ses collègues dans les termes suivants:

Messieurs, vous connaissez tous l'extrême difficulté que les préfets éprouvent à trouver des maires dans nos campagnes [...]. Comment un préfet [...] pourra-t-il y parvenir dans plusicurs communes où l'on compte à peine cinq à dix personnes qui savent écrire et dont aucune n'a le degré d'instruction nécessaire pour comprendre le langage des lois? $?^{20}$

Dès lors, les relations entre l'État et les communes n'ont pu qu'évoluer mais sans doute plus rapidement que les représentations qui leur sont associées.

\section{La crise du modèle hiérarchique des relations entre l'État et les communes: une décentralisation en quête de reconnaissance}

L'échec, relatif mais patent, de l'action de l'État à partir des années 1970 rencontre l'aspiration à une autonomie plus forte des localités pour atteindre la légitimité du modèle hiérarchique des relations entre l'État et les communes. Ia décentralisation de 1982 n'a fait qu'amorcer un mouvement d'adaptation de l'action territoriale de l'État qui bute encore, même en 2004, sur les traditionnelles représentations jacobines. Pour aller plus loin, il semble nécessaire de les remettre en question sur le plan des représentations et de reconnaitre juridiquement et politiquement les communes dans un nouveau rôle qu'elles ont déjà, dans la pratique, commencé à construire.

${ }^{20}$ Déclaration du député Fleuriau de Bellevue, le 4 févtier 1831, cité par Chandernagor (1993: 186). 


\subsection{La crise du modèle hiérarchique de l'action territoriale de l'État}

En dépit d'évolutions significatives, telles que les grandes lois départementale de 1871 et municipale de 1884, de l'apparition modeste et ponctuelle d'un « socialisme municipal » ${ }^{21}$, les relations entre l'État et le territoire demeurent marquées par «l'habitus » centralisateur qui accentue, sous la période gaulliste de la $V^{e}$ République, son caractère technocratique. Nous connaissons l'attachement de l'homme du 18 juin 1940 à l'État permettant, selon lui, et de manière quelque peu martiale, au pays « sous peine de danger mortel, [de ] viser haut et [ de ] se tenir droit » (de Gaulle,1954: 1). Son hostilité, notamment au début de la $V^{\mathrm{e}}$ République, à la classe politique notabiliaire incarnant par trop les mours parlementaires des régimes passés n'est pas non plus un mystère. Cette double inclination associée à la volonté de « modemiser $~_{22}$ le pays induira des politiques volontaristes - aménagement du territoire, politiques sectorielles et rationalisation administrative - caractérisées par une même inspiration technocratique. Nous pouvons parler avec Habermas (1978) du développement d'une « conscience technocratique » qui conduit à « l'élimination de la différence entre pratique et technique ». Avec elle, un modèle de décision de portée universelle s'impose. Il s'agit de traiter tout problème pratique, de l'aménagement urbain aux politiques sectorielles par exemple, en le ramenant à un algorithme technique niant, de la sorte, les dimensions proprement politiques des choix. La centralisation technocratique légitimée par la croissance des «trente glorieuses » va pâtir de son brusque ralentissement durant la décennie 1970 et nombre de critiques se font jour à la faveur de l'incapacité de l'État à garantir « la Providence ». Sur le plan de l'administration territoriale, les diagnostics se succèdent pour décrire, à l'instar de la commission Guichard (1976), « une présence obsédante de l'État », « sa prolifération réglementaire ", l'inefficacité de « la juxtaposition de ses structures verticales » et l'inadaptation des territoires institutionnels aux réalités socio-économiques. Ces figures de l'échec ouvrent un espace pour le développement de thématiques jusque là marginales telles que le développement territorial et l'autonomie, voire l'identité locale. Ces nouveaux enjeux correspondent aux nécessités économiques - les carences du centre - et aux aspirations sociopolitiques d'une population de plus en plus éduquée et

21 À la croisée de la "question sociale " et de la "question urbaine ", J.-P. Gaudin, Technopolis, crises urbaines et innovations saciales, Paris, PUF, 1989.

${ }^{22}$ L'un des mots les plus usités dans la thétorique gaullienne. 
désireuse, pour partie, de prendre des responsabilités publiques ${ }^{23}$. De manière congruente, les "nouveaux mouvements sociaux » décrits par Touraine (1973) portent une critique active de la technocratie.

L'Etat, dans ces conditions, ne peut plus régenter le territoire ou continuer d'apparaitre comme son tuteur. Les maires des grandes villes contestent la tutelle et entrent dans des conflits ouverts avec l'autorité préfectorale comme Defferre à Marseille à propos du rachat par la ville des chantiers navals Terrin. Le cadre légal fixant les rapports entre les collectivités territoriales et l'État apparaît de plus en plus contesté. La décentralisation de 1982 peut alors apparaître comme une forme de réponse à cette situation. Mais a-t-elle, pour autant, réalisé la "réforme psychologique » souhaitée dans le rapport Guichard ou encore «le renouvellement de méthode intellectuelle» préconisé par Crozier (1971: 218)?

\subsection{La décentralisation « au milieu $d u g$ gé » ou la résistance des traditionnelles représentations jacobines}

La décentralisation constitue de toutes les réformes initiées par Mitterrand " celle dont les effets ont été immédiats, profonds et irréversibles " (Colombani et Portelli, 1995: 67). Elle met fin au cycle biséculaire de centralisation en créant à la marge les conditions d'un processus décentralisateur par appropriation par les collectivités territoriales de nouveaux pouvoirs. La dynamique ainsi créée a produit un indiscutable renouvellement et une stimulation de l'action publique locale. En favorisant l'autonomie locale, elle tente de rompre avec le modèle hiérarchique qui prévalait jusque là. Mais un bref retour sur le processus politique de sa mise en cuvre montre que cette réforme doit beaucoup à son initiateur, Defferre.

\subsubsection{La réforme de 1982: un changement « à la bussarde » du modèle biérarchique}

Conscient de la grande capacité de reproduction du système centralisé, il rompt avec «le réformisme précautionneux $»^{24}$ du gouvemement antérieur et mène dans l'urgence une stratégie de rupture. Il prend personnellement en

\footnotetext{
${ }^{23}$ Ce qui s'est manifestée notamment aux élections municipales de 1977.

${ }^{24}$ C'est ainsi que F. Mitterrand, alors premier secrétaire du parti socialiste, stigmatisait à l'Assemblée nationale les projets prudents et modestes de décentralisation défendus par le ministre de l'Intérieur du gouvernement Barre, R. Bonnet.
} 
charge la rédaction du projet de loi, en s'entourant de quelques proches et en rejetant la participation des hauts fonctionnaires ${ }^{25}$. Comme l'indique Quillot, « il marcha au canon [...], bouscula le conseil des ministres et imposa ses vues à la hussarde jusqu'à la promulgation de la loi du 2 mars » (Le Monde, 4 mars 1977). Sans cette précipitation, il n'est pas interdit de penser, dans la perspective d'une «histoire hypothétique ", que les mauvais résultats de la majorité aux scrutins locaux dès 1982 auraient pu réveiller l'hostilité de l'aile jacobine du parti socialiste et autoriser une résistance plus efficace de la haute fonction publique pour finalement enliser cette réforme disparaissant de l'agenda politique au profit des questions économiques. La réussite de cette stratégie du coup de force ne doit donc pas laisser accroire que sur les plans idéologique et cognitif la bataille de la décentralisation était gagnée.

Certaines réactions préfectorales témoignent de la prégnance de la conception hiérarchique des relations entre l'État et les collectivités territoriales. Examinons celle, il est vrai particulièrement vive, du préfet déjà cité, Vié en lui appliquant un modèle d'analyse psychosociologique. Toute représentation, ainsi que l'a montré Abric (1994), est organisée autour d'un noyau ou système central, producteur de la signification et de l'organisation de la représentation, et d'un système périphérique dont la plasticité permet l'adaptation. Ici le noyau central se structure autour des principes de légalité, d'unité, d'égalité et de centralité pour déboucher sur le modèle hiérarchique des relations entre le préfet et le territoire. Si les allégements successifs de la tutelle ont pu être intégrés au système périphérique de la représentation sans mettre en péril le noyau central, sa suppression en 1982 enclenche un processus, plus ou moins rapide mais inéluctable, d'adaptation de l'ensemble de la représentation auquel certains échappent en refusant d'admettre la décentralisation. C'est le cas de l'ancien préfet cité. Le processus d'intégration d'un schème étrange dans une représentation suit, selon Flament (1994), la séquence suivante: rappel du normal, désignation de l'élément étranger, affirmation de la contradiction entre les deux termes, et proposition d'une rationalisation permettant de surmonter la contradiction. Dans son ouvrage La décentralisation sans illusion (1982), Vié commence par rappeler le normal, c'est-à-dire la centralisation avec son contrôle a priori résultant " d'une collaboration confiante » entre les élus et les préfets et produisant des résultats

\footnotetext{
${ }^{25}$ Auxquels il aurait déclaré, selon R. Quillot associé avec M. Charasse à la rédaction du texte: "Rentrez dans vos bureaux nous nous débrouillerons sans vous! ", ( La décentralisation reste une cathédrale inachevée ", Le Monde, 4 mars 1997).
} 
efficaces. Puis vient la désignation de l'élément étranger: la décentralisation et la fin de la tutelle ayant des conséquences désastreuses - «impossibilité pour l'État de faire prévaloir ses décisions autrement que par la loi »; « incapacité », "manque de formation et... de goût esthétique (sic) » des élus; et enfin, contrairement «à un préfet qu'on change s'il n'obéit pas » il faudra demain «si l'élu n'obéit pas, composer avec lui et patienter jusqu'à ce que le tribunal administratif ou le Conseil d'État le ramène au respect de la loi ». Il y a donc bien contradiction entre le normal et cet élément étranger. Comment est-elle résolue? De la manière suivante: « Si la France est appelée à poursuivre sa destinée, il faudra que le balancier de l'histoire, dans ce domaine comme dans beaucoup d'autres, revienne largement en arrière » (Vié, 1982: 138). Il s'agit bien d'un refus d'accepter la décentralisation et la fin du préfet tuteur au nom d'une représentation hiérarchique, ici inébranlable.

Insupportable à plus d'un préfet, la décentralisation initiée par Defferre modifie le système politico-administratif avec une grande habileté stratégique qu'on pourrait croire issue de la connaissance de la sociologie des organisations.

\subsubsection{La conception sociologique de l'administration locale}

Tout comme la sociologie du travail s'est construite sur l'analyse et la critique des principes tayloriens et fordiens, la sociologie des organisations publiques locales s'est développée contre l'omnipotence de la vision juridique de ces organisations.

Dans un article paru dans Espriten 1970, Worms et Grémion, après avoir décrit le fonctionnement du système politico-administratif local qui sera popularisé par Le pouvoir périphérique (Grémion, 1976), préconisaient quatre types d'action: la clarification des compétences, la mise en responsabilité des acteurs, l'acceptation du fait que le pouvoir est partagé et partenarial et, enfin, le renversement de l'initiative au profit du terrain. La seule stratégie possible, selon eux, était de « casser » ce système autorégulé d'administration locale.

Cette analyse inspira la démarche appliquée par Defferre en 1981. En fait, en supprimant la tutelle, la décentralisation ne modifiait que certaines règles du jeu local sans « remettre à plat » le système politico-administratif territorial ni tenter de le reconstruire globalement. Et, effectivement, la question du découpage municipal est soigneusement contoumée. De même, nous ne choisissons pas entre le département et la région. Avec une vraie sensibilité sociologique, Defferre sait que les enjeux de telles questions sont très forts et 
risquent de faire échouer la réforme. Aussi décide-t-il de ne modifier que les règles du jeu, mais de manière suffisante pour que le jeu en soit réellement changé. Comme l'écrit Thoenig, il crée « une mutation suffisante pour laisser passer un nouveau jeu, en le rendant vite irréversible tout en ne le figeant pas, la porte restant ouverte à une dynamique de l'appropriation par le terrain » (Thoenig, 1999: 95). Le changement du système d'action local s'est donc fait sans bouleversements institutionnels et, engendrantà la fois plus d'autonomie et plus de responsabilisation de la part des élus, il en a changé le comportement: le notable s'est transformé en gestionnaire.

C'est donc à partir d'une modification « incrémentale » (ibid.) du système d'action - ici la suppression de la tutelle - qu'une dynamique endogène de changement se met en cuvre. La connaissance formelle, au demeurant assez probable, de la sociologie des organisations par Defferre n'est d'ailleurs pas nécessaire; l'essentiel est de montrer que le raisonnement organisationnel inspire, d'une manière ou d'une autre, la mise en œuvre de la décentralisation. Il reste maintenant à savoir si, comme nous le pensons, la représentation des relations État-communes véhiculée par la sociologie des organisations, ou, aujourd'hui, de l'action organisée, peut être caractérisée comme plus complexe que la représentation traditionnelle d'inspiration politico-juridique.

Dans le modèle traditionnel hérité des Lumières et de la Révolution, la commune est hiérarchiquement subordonnée à l'État qui possède le pouvoir. Dans ce rapport univoque de subordination se donne à voir l'orientation mécaniste des représentations du monde en même temps qu'un véritable culte de l'autorité. Décidé au sommet de l'État pour s'appliquer aux tréfonds du corps social, le pouvoir descend, tel le «fluide électrique » dans un impeccable ordonnancement politico-administratif dont la rigueur répétitive et la rentabilité instrumentale évoquent en tous points le modèle de la loi physique.

À cette représentation linéaire et univoque du monde politico-administratif, la sociologie des organisations a opposé l'interdépendance du système. Le pouvoir y apparaît de nature relationnelle et non pas substantielle. Autrement dit, à l'injonction se substituent la négociation et, plus généralement, le jeu.

Au caractère abstrait de la représentation politico-juridique s'oppose le caractère concret de la vision sociologique. Dans la première, en vertu du principe de légalité, la norme juridique s'impose uniformément aux hommes. C'est la loi qui ordonne au monde social dans une sorte de déterminisme juridique. Ainsi le législateur révolutionnaire institua-t-1l, en 1789, 44000 communes au statut identique parmi l'infinie variété des formes communau- 
taires existantes. Dans la seconde, la règle n'est importante qu'en tant que règle en action, c'est-à-dire mise en œuvre par les acteurs. Elle constitue un élément, ressource ou contrainte selon les acteurs, qui n'existe concrètement que dans et par le « construit social» formé par les interrelations entre les différents acteurs. Toujours médiatisée par le jeu, la règle formelle ne saurait suffire à décrire les organisations - État ou communes - et les relations entre elles. Même si elle n'exclut pas, dans son fonctionnement quotidien, les régularités, une organisation, parce qu'elle est toujours le résultat de l'action de ses membres, ne saurait être enfermée dans un déterminisme mécanique. Avec la conception sociologique de la représentation des relations Étatcommunes nous sortons de la métaphore si prégnante de la machine pour admettre l'incertitude.

Enfin, pour le sociologue de l'organisation, rétif aux généralisations nomothétiques et aux constructions abstraites, le monde de l'organisation doit être saisi concrètement dans la variété de ses formes permise par l'autonomie, même relative, des acteurs. Il n'est pas d'uniformité qui ne relève de l'abstraction. En d'autres termes, à l'uniformité politico-juridique, postulée abstraitement, des communes et de leurs relations avec l'État, il faut substituer la diversité des systèmes d'action saisis concrètement.

Mais pour l'heure, constatons que la représentation des relations Étatcommunes d'inspiration sociologique apparait moins hiérarchique, moins déterministe, et moins uniforme que la représentation politico-juridique. Fondée sur l'interdépendance, admettant l'incertitude et la diversité, elle tend vers plus de complexité. Ainsi, en s'inspirant en conscience ou, comme Monsieur Jourdain, de la représentation de type sociologique, Defferre travaillait aussi à la construction d'un monde local plus complexe.

\subsubsection{Une certaine remise en cause des principes anciens: essai de schématisation des} fondements idéologiques et cognitifs de la représentation associée à la décentralisation de 1982

La décentralisation, non remise en cause par les alternances politiques, s'inscrit durablement dans les faits et implique un amendement des fondements idéologiques et cognitifs présidant à l'action territoriale de l'État. Parmi les quatre principes fondateurs - légalité, unité, égalité et centralité - c'est le dernier qui voit son influence reculer même si une cohérence « jacobine» demeure forte entre les trois premiers. 
Rokkan (1975) a montré que la France avait connu un développement politique monocéphal, ce que confirment, sans surprise, Badie et Birnbaum en évoquant à son propos de «l'ideal-type de l'État unitaire centralisé » (1982). Cependant, ce monocentrisme traditionnel français cède, peu à peu, à un polycentrisme de fait tant sur le plan politique avec la construction européenne que sur le plan économique avec la mondialisation. De plus, le modèle hiérarchique régenté du centre, de plus en plus rejeté dans la gestion des organisations productives, n'est plus culturellement accepté par les collectivités territoriales. L'autonomie, même relative, accordée aux collectivités par la décentralisation crée les conditions d'une moindre prégnance du centre et l'émergence d'un timide polycentrisme sur le territoire national. Le développement de la contractualisation des politiques publiques et l'injonction faite aux territoires de définir des projets en sont des signes.

Le recul de la centralité et l'augmentation de l'influence du principe d'autoorganisation sont les deux grandes évolutions idéologiques de la représentation associée à la décentralisation de 1982. Nous ne pouvons méconnaittre ici l'influence du libéralisme qui gagne l'Occident dans les années 1980. D’ailleurs, la Banque mondiale ne préconise-t-elle pas comme modes d'adaptation des sociétés à la mondialisation, la privatisation et ... la décentralisation? De fait, dans la tradition française, un courant libéral incarné par Tocqueville, a, depuis longtemps, critiqué la centralisation après que le court épisode de 1789-1791 se soit soldé par la défaite des Girondins. Les origines politiques de la décentralisation de 1982 sont ailleurs - le courant autogestionnaire et le régionalisme d'après-guerre - mais elles partagent avec les libéraux la critique du principe de centralité.

Constatons la congruence de cette vision idéologique avec le développement dans divers champs scientifiques de théories qui rompent avec la conception « horlogère » du monde issue de la mécanique newtonienne: la physique quantique qui est, certes, bien antérieure mais dont la vulgarisation sera beaucoup plus tardive, la théorie des structures dissipatives et de l'ordre par fluctuation de Prigogine, l'auto-organisation dans la biologie parmi d'autres. Ces théories décrivent un monde moins déterminé donc moins prévisible, où l'ordre émerge du désordre pour y retourner et où les organismes vivants sont capables d'une complexification endogène qui leur confère une plus grande autonomie dans leur environnement. Ces conceptions scientifiques commencent à irradier l'ensemble des représentations sociales y compris celle concernant les relations État-collectivités territoriales. 


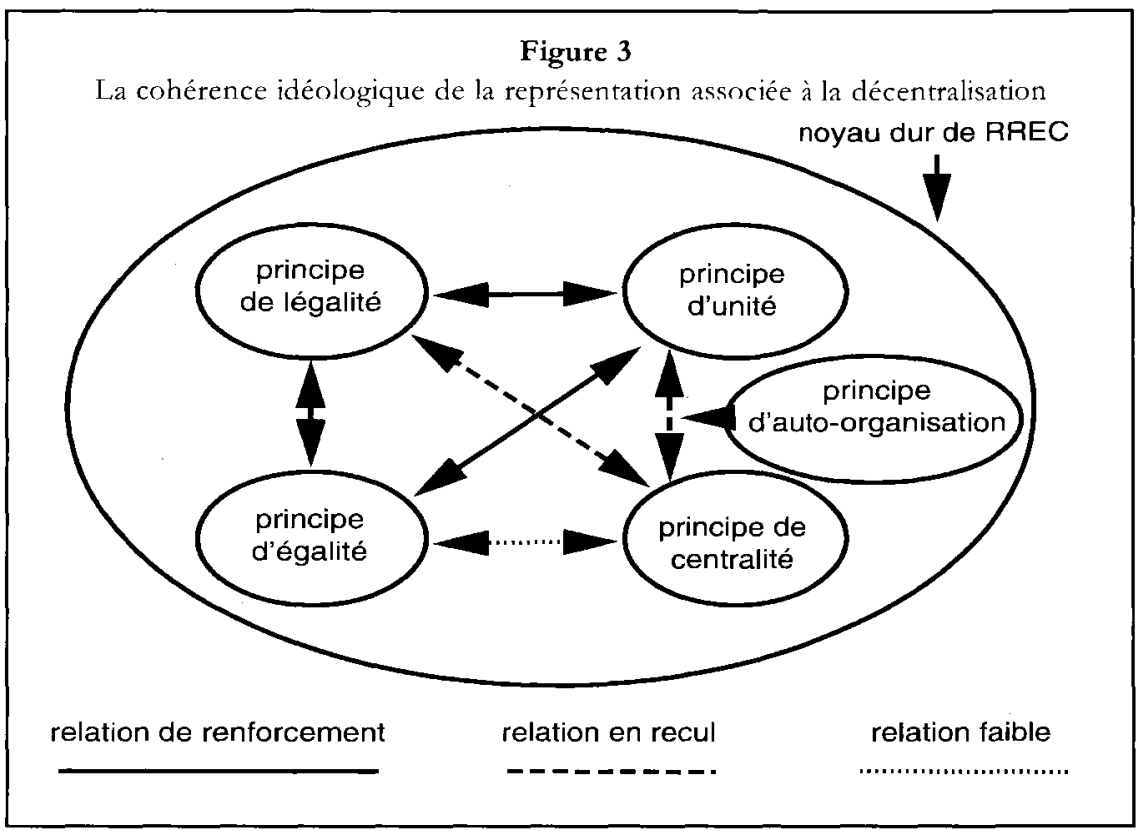

Figute 4

La cohérence cognitive de la représentation attachée à la décentralisation

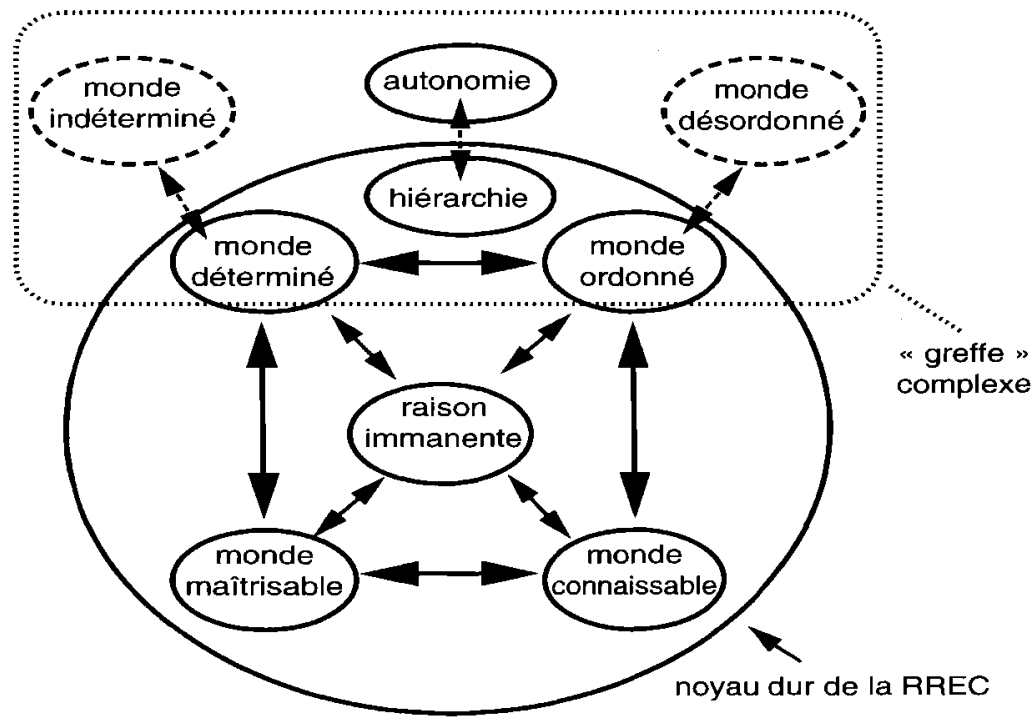


Cette évolution lente des représentations s'est accompagnée d'un changement plus rapide des pratiques dont témoignent les changements qui ont affecté le rôle du préfet et qui tendent à une forme de complexification.

\subsection{Une recomposition néanmoins, celle du rôle préfectoral sur le territoire}

Perdant l'exercice des exécutifs départementaux et régionaux et le contrôle $a$ priori des actes des collectivités territoriales, le préfet a vu, douloureusement quelquefois, comme nous l'avons vu, sa capacité d'action se réduire notamment dans ses relations avec les élus locaux ${ }^{26}$. Il ne dispose plus du même ascendant hiérarchique sur la scène locale et apparaît rendu au rang d'acteur parmi d'autres ${ }^{27}$, devant construire une autorité qui lui était, par le passé, accordée d'entrée de jeu. Le faible nombre des déférés préfectoraux témoigne d'ailleurs que les préfets sont « très soucieux de la qualité de leurs relations avec les élus et peu motivés par la saisine du juge » (Hélin et Hostiou, 1996: 335). De fait, leur rôle a changé et les préfets s'y sont adaptés. Le préfet Bemard décrivait, en 1992, l'« art préfectoral »(Bernard, 1992: 133-144) en utilisant des termes révélateurs: " partage », « ajustement », " ouverture », " magistrature de médiation », " concertation » ou encore " coopération ». Avec la décentralisation, le préfet se fait animateur et médiateur parmi les acteurs du territoire tout en représentant l'intérêt national. La configuration n'est plus celle « du préfet et ses notables » (Worms, 1966) où le premier avait la haute main mais celle d'un jeu plus équilibré entre le représentant de l'État sur le territoire et les représentants locaux du territoire.

À certains égards, cette pratique coopérative pourrait annoncer l'émergence d'un nouveau type de droit, car, comme l'écrit le sociologue du droit Willke, «non seulement le droit traditionnel, mais aussi le principe de légalité sont trop simples pour être capables d'offrir des solutions à ces problèmes profonds issus des sociétés modemes » (1992: 136). Ce qu'il appelle " des programmes relationnels » permettraient, selon lui, « l'autorégulation par les parties et, en même temps, la transformation des processus autorégulateurs en buts communs librement consentis » (Willke, 1991: 85). En d'autres termes, ce droit réflexif, pourrait autoriser une autorégulation des acteurs locaux définissant un

\footnotetext{
${ }^{26}$ Grands et moyens dans la typologie de Mabileau (1991).

${ }^{27}$ En 1991, $57 \%$ des membres du corps préfectoral se sentaient acteurs contre 38 arbitres (P. Bernard, 1992: 202).
} 
projet, le représentant de l'État intervenant non comme arbitre mais comme médiateur des intérêts nationaux. Ainsi, les acteurs locaux pourraient être associés à la définition de la règle qui leur serait applicable dans le cadre d'un processus discursif prenant en compte, à la fois, les intérêts locaux et nationaux. Une telle mutation de la légalité ne manquerait pas de concerner aussi les principes d'unité et d'égalité.

Si la complexité des pratiques concrètes défie et dément toujours la simplicité des prescriptions et des représentations, ces dernières constituent néanmoins des contraintes pour l'action. Leur évolution peut, dès lors, permettre un renouvellement de l'action. Or, les relations entre l'État et les collectivités locales demeurent encore codifiées par des dispositifs juridiques s'inspirant d'une conception uniforme de l'unité nationale et une vision abstraite de l'égalité. Pourtant, des portes ont été entrouvertes en 2003. Ainsi, par exemple, le droit à l'expérimentation accordé, sous contrôle parlementaire, aux collectivités territoriales et surtout le «déverrouillage » constitutionnel du principe d'identité institutionnelle qui prohibait toute évolution statutaire significative des collectivités territoriales ont été reconnus lors de la réforme constitutionnelle de 2003. Reste, en cas de diversification institutionnelle, et ce n'est pas une mince affaire, à garantir, d'une part, une égalité de traitement entre les situations identiques et, d'autre part, à assurer aux collectivités territoriales moins dotées de ressources la possibilité de profiter de la solidarité nationale. Nous mesurons là que le rôle du préfet apparait tout à fait essentiel. En effet, dans le cadre d'une unité choisie plus qu'imposée, d'une légalité plus négociée et d'une égalité plus attentive aux situations concrètes, le préfet représenterait le partenaire, à la fois légitime et puissant, incarnant les intérêts nationaux dans des dynamiques territoriales renouvelées et diversifiées et agissant plus sur le mode de l'incitation et de la négociation que sur celui de l'autorité supérieure contraignante.

De leur origine révolutionnaire et napoléonienne les relations entre l’État et les communes ont longtemps conservé la marque. Instituteur et contrôleur du territoire, l'État en assurait le caractère unitaire par l'application égalitaire de la loi nationale. L'homologie avec une conception horlogère du monde issue de la science classique conférait à son action, et plus généralement à celle de l'État, la légitimité de la Raison en marche vers le Progrès. C'est par cette « ceuvre de civilisation » que l'État a institué la Nation. Mais, le succès de cette entreprise portait en germe la contestation des conditions qui l'ont permis. Une société plus éduquée, plus dynamique et plus mobile aspirait à plus d'autonomie. À la faveur du démenti apporté à l'omnipotence de l'État par 
les décennies postérieures à 1970, le modèle hiérarchique des rapports Étatcollectivités territoriales s'est amendé avec la décentralisation. Le rôle concret de l'État et de son représentant local, le préfet, s'est transformé sans que la " cohérence jacobine » des principes de légalité, d'unité et d'égalité soit réellement remise en question. Cette permanence contribue à expliquer pourquoi la décentralisation est longtemps demeurée « au milieu du gué » avant de connaître une « seconde vague » dans l'indifférence, voire l'hostilité. Pourtant, dans un monde que de nouvelles théories scientifiques décrivent comme étant plus incertain, moins déterminé et où l'autonomie organisationnelle apparait l'une des clefs du vivant, doit-on continuer à concevoir l'unité comme synonyme d'uniformité et l'égalité comme isonomie?

\section{Références}

Abric, Jean-Claude (1994), « L’Organisation interne des représentations sociales : système central et système périphérique ". In: Christian Guimelli (dir.), Structures et transformations des représentations sociales. Neuchâtel, Delachaux et Niestlé, 73-84.

Agulhon, Maurice (1979). La Démocratie au village. Les populations du Var de la Révolution à la $I I^{e}$ République. Paris, Le Seuil.

Amaud, André-Jean (1992). « Du jeu fini au jeu ouvert, vers un Droit post-moderne ». In: François Ost et Michel Van de Kerchove (dir.), Le jeu, un paradigme pour le droit. Paris, LGDJ, coll. Droit et Société, 111-131.

Badie, Bertrand et Birnbaum, Pierre (1982). Sociologie de l'État. Paris, Grasset.

Baverez, Nicolas (2003). La France qui tombe. Paris, Perrin.

Bernard, Paul (1992). Le Préfet de la République. Le chêne et l'olivier. Paris, Economica.

Berthelot, Jean-Michel (1990). L'Intelligence du social. Paris, PUF.

Bredin, Jean-Denis (1988). Sieyès, La clé de la Révolution française. Paris, de Fallois.

Bourdieu, Pierre (1997). Méditations Pascaliennes. Paris, Liber.

Bourjol, Maurice (dir.)(1990). La Commune, l'État et le droit. Paris, LGDJ.

Burke, Edmund (1912). Réflexions sur la Révolution française. Paris, Nouvelle Librairic Nationale, [1790].

Cassirer, Ernst (1970). La Philosopbie des Lumières. Paris, Fayard, [1932].

Chandernagor, André (1993). Les Maires de France, XIX ${ }^{e}-X X^{e}$. Paris, Fayard.

Cohen-Tanugi, Laurent (1989). La Métamorphose de la démocratie. Paris, O. Jacob.

Colombani, Jean-Marie et Portelli, Hugues (1995). Le double septennat de Francois Mitterrand. Paris, Grasset.

Crozier, Michel (1971). La Société bloquée. Paris, Seuil.

De Gaulle, Charles (1954). L'Appel. Mémoires de Guerre. Paris, Plon.

De Maistre, Joseph (1988). Considérations sur la France. Essai sur le principe générateur des constitutions politiques. Paris, Complexe, [1936 ].

Deyon, Pierre (1996). L_État face au pouvoir local. Paris, Éd. Locales de France. 
Dilthey, Wilhem (1942). Théorie des conceptions du monde. Paris, PUF, [1911].

Donzelot, Jacques (1984). L'Invention du social. Paris, Fayard.

Favoreu, Louis (1983). «Les Bases constitutionnelles du droit des collectivités territoriales».

La nouvelle décentralisation. Paris, Sirey, 15-37.

Flament, Claude (1994). "Structure, dynamique et transformation des représentations sociales m. In: Jean-Claude Abric (dir.), Pratiques sociales et représentations. Paris, PUF, 37-57.

Garcia, Enterria Eduardo de (1995). Révolution frangaise et administration contemporaine. Paris, Economica, [1994] .

Gaudin, Jean-Paul (1989). Technopolis, crises urbaines et innovations sociales. Paris, PUF.

Gazette nationale ou Le Moniteur universel (1789). $\mathrm{n}^{\text {os }} 65$ (p. 70 ), 74 (p. 71 ) et 79 (p. 69).

Gremion, Pierre (1976). Le Ponvoir périphérique. Paris, Le Seuil.

Groethuyssen, Bernard (1956). Philosopbie de la Révolution frangaise. Paris, Gonthier.

Guichard, Olivier (1976). Viure ensemble. Rapport de la commission de développement des responsabilités locales. Paris, La documentation française.

Habermas, Jürgen (1978). La Tecbnique et la science comme « idéologie ». Paris, Gallimard, [1968].

Hélin, Jean-Claude et Hostiou, René (1996). "Réactiver le juge administratif ». La décentralisation en France. Paris, La découverte, 329-343.

Jobert, Bruno et Muller, Pierre (1987). L'État en action. Paris, PUF.

Laflamme, Simon (2000). Des Biens, des idées et des personnes au Canada (1981-1995): un modèle macrologique. Paris-Sudbury, L'Harmattan-Prise de parole.

Latour, Bruno (1989). La Science en action. Paris, La découverte.

Le Moigne, Jean-Louis (1995). Les Épistémologies constructivistes. Paris, PUF, coll. QSJ.

Le Monde (1997). «La Décentralisation reste une catbédrale inachevée ». 4 mars.

Le Moniteur universel ou Gazette nationale (1789). $\mathrm{n}^{\text {os }} 65$ (p. 70 ), 74 (p. 71 ) et 79 (p. 69).

Mabileau André (1991). Le Système politique local. Paris, Montchrestien.

Maurras, Charles (1948). L'ordre et le désordre. Les idées positives et la Révolution. Paris, Self.

Morin, Edgar (1977). La Méthode. Tome 1, «La nature de la nature ». Paris, Seuil.

Ohnet, Jean-Marie (1996). Histoire de la décentratisation française. Paris, Le Livre de Poche.

Ozouf-Marignier, Marie-Vic (1989). La Formation des départements. La représentation du territoire à la fin du XVIII ${ }^{e}$ siècle. Paris, Éd. de l'EHESS.

Panofsky, Erwin (1967). Architecture gothique et Pensée scolastique. Paris, Éd. de Minuit, [1951].

Rawls, John (1987). Théorie de la justice. Paris, Seuil.

Roggero, Pascal (1997). Des Communes dans la complexité: représentations et politiques. Thèse de sociologie, Université de Toulouse 1.

Roggero, Pascal (2002). «Le Préfet entre hiérarchie et complexité ». Jean-Pierre Allinne (dir.), Le Préfet et le développement local. Paris, Dalloz, 171-184.

Rokkan, Stein (1975). "Dimension of State Formation and Nation Building ». In: Charles Tilly. The Formation of National States in Western Europe. Princeton, Princeton University, 562-600.

Rosanvallon, Pierre (1981). La Crise de l'État providence. Paris, Le Seuil.

Rosanvallon, Pierre (1992). Le Sacre du citoyen. Histoire du suffrage universel en France. Paris, Gallimard, Bibliothèque des histoires.

Rosanvallon, Pierre (1998). Le Peuple introuvable. Histoire de la représentation démocratique en France. Paris, Gallimard, Bibliothèque des histoires.

Rosanvallon, Pierre (2000). La Démocratie inacbevée. Histoire de la souveraineté du peuple de France.

Paris, Gallimard, Bibliothèque des histoires. 


\section{NPSS, VOLUME 1, NUMÉRO 1, 2005}

Rosanvallon, Pierre (2002). "Histoire moderne et contemporaine du politique ». Site consulté en septembre 2004: http://www.college-de-france.fr/media/ his pol/LPL521. 10 RosanvallonR0102.pdf.

Rosanvallon, Pierre (2004). Le Modèle politique francais. La société civile contre le jacobinisme de 1789 à nos jours. Paris, Le Seuil.

Ruelle, Olivier (1993). « Le Légicentrisme républicain ». In: François Furet et Mona Ozouf (dir.), Le Siècle de l'avènement républicain. Paris, Gallimard, 441-471.

Sfez, Lucien (1989). L'Égalité. Paris, PUF, Coll. QSJ.

Thoenig, Jean-Claude (1999). "L'Usage analytique du concept de régulation ». In: J. Comaille et $\mathrm{P}$. Jobert (dir.), Les métamorphoses de la régulation juridique. Paris, LGDJ.

Thouret (1789). Rapport de Thouret. 29 septembre.

Touraine, Alain (1973). Production de la société. Paris, Seuil.

Tulard, Jean (1985). «Les Révolutions, de 1789 à 1851 ». In: Jean Favier (dir.), Histoire de France, Paris, Fayard.

Vié, Jean-Emile (1982). La Décentralisation sans illusion. Paris, PUF.

Vié,Jean-Emile (1997). «Impudence ou inconscience ». Revue administrative, n 297, 308-309.

Weber, Eugen (1983). La Fin des terroirs. La modernisation de la France rurale 1870-1914. Paris, Fayard.

Willke, Helmut (1991). «Trois types de structures juridiques: programmes conditionnels, programmes finalisés et programmes relationnels ». In: Charles-Albert Morand (dir.), L'État-propulsif, contribution à l'étude des instruments d'action de l'État. Paris, Publisud, 65-94.

Willke, Helmut (1992). «Le Coût du principe de légalité ». In: Charles-Albert Morand (dir.), Figures de la légalité. Paris, Publisud.

Worms, Jean-Pierre (1966). "Le Préfet et ses notables ». Revue franģaise de sociologie, 8 (3), 249276.

Worms, Jean-Pierre (1990). "La Décentralisation au milieu du gué ». Revue politique et parlementaire, 946, 11-19.

Worms, Jean-Pierre et Grémion, Pierre (1970). «L'État et les collectivités locales ». In: Esprit, 1, 335-544. 